\title{
An electron microscopy study of microstructural evolution during in-situ annealing of heavily deformed nickel
}

\author{
Zhang, Yubin; Yu, Tianbo; Mishin, Oleg
}

Published in:

Materials Letters

Link to article, DOI:

10.1016/j.matlet.2016.09.082

Publication date:

2017

Document Version

Peer reviewed version

Link back to DTU Orbit

Citation (APA):

Zhang, Y., Yu, T., \& Mishin, O. (2017). An electron microscopy study of microstructural evolution during in-situ annealing of heavily deformed nickel. Materials Letters, 186, 102-104.

https://doi.org/10.1016/j.matlet.2016.09.082

\section{General rights}

Copyright and moral rights for the publications made accessible in the public portal are retained by the authors and/or other copyright owners and it is a condition of accessing publications that users recognise and abide by the legal requirements associated with these rights.

- Users may download and print one copy of any publication from the public portal for the purpose of private study or research.

- You may not further distribute the material or use it for any profit-making activity or commercial gain

- You may freely distribute the URL identifying the publication in the public portal

If you believe that this document breaches copyright please contact us providing details, and we will remove access to the work immediately and investigate your claim. 


\title{
An electron microscopy study of microstructural evolution during in-situ annealing of heavily deformed nickel
}

\author{
Y.B. Zhang*, T. Yu, O.V. Mishin \\ Section for Materials Science and Advanced Characterization, Department of Wind Energy, \\ Technical University of Denmark, Risø Campus, Roskilde 4000, Denmark \\ *Corresponding author: yubz@dtu.dk
}

\begin{abstract}
The microstructure of heavily deformed pure nickel processed by accumulative roll bonding to a von Mises strain of 6.4 has been investigated using both transmission electron microscopy and transmission Kikuchi diffraction in a scanning electron microscope. By monitoring the microstructure in one region during in-situ annealing in a transmission electron microscope, it is found that $9 \%$ of all triple junctions present in this region have migrated over more than $40 \mathrm{~nm}$. Junctions formed by three high angle boundaries are observed to be more prone to motion during recovery than any other junctions. The extent of triple junction motion in the Ni sample is compared to that in heavily deformed aluminum.
\end{abstract}

Keywords: electron microscopy; transmission Kikuchi diffraction (TKD); nickel; microstructure; recovery; triple junction motion

\section{Introduction}

Nanocrystalline metals produced by severe plastic deformation (SPD) are characterized by a very high stored energy, which makes their microstructure unstable. For example, static recrystallization has been observed in heavily deformed copper and silver after room temperature (RT) storage [1-3]. The deformed microstructure may also coarsen during recovery by migration of triple junctions (TJs), as shown for aluminum annealed after SPD [4-8]. In contrast to heavily rolled Al, where lateral motion of Y-junctions takes place even at RT [4], no evidence of such motion has been found after long-term RT storage of nickel processed by accumulative rolling 
bonding (ARB) to a von Mises strain $\varepsilon_{\mathrm{vM}}=4.8$ [9]. Apparently, the energy stored during ARB in this Ni sample was insufficiently high to trigger structural coarsening at RT.

In the present work, we investigate $\mathrm{Ni}$ deformed by ARB to a much higher strain, $\varepsilon_{\mathrm{vM}}=6.4$, which resulted in a finer boundary spacing [10] and thus in a higher stored energy. Microstructural changes in this material are monitored during in-situ annealing in a transmission electron microscope (TEM), aiming at establishing whether TJ motion takes place in heavily deformed Ni. Additionally, a new scanning electron microscope (SEM)-based characterization technique, termed transmission Kikuchi diffraction (TKD) [11], is utilized to measure boundary misorientation angles before annealing. TKD allows a better spatial resolution than standard EBSD, and is well suited to studies of nanocrystalline materials [12-14]. The microstructural evolution during recovery in this heavily strained Ni sample is compared to that in Al deformed to similar strains.

\section{Experimental}

The material chosen for this work was pure (99.967\%) nickel processed by ARB to $\varepsilon_{\mathrm{vM}}=6.4$ [10]. A thin foil was prepared from the longitudinal section containing the normal and rolling directions (ND - RD). At first, the microstructure was investigated by TKD in a Zeiss Supra 35 SEM. In the TKD experiment, the foil was tilted to $-5^{\circ}$ and crystallographic orientations were measured at a working distance of $5 \mathrm{~mm}$ and an accelerating voltage of $30 \mathrm{kV}$. In this foil, a region with an area of $\sim 16 \mu^{2}$ was mapped near the mid-thickness of the ARB sample using a step size of $10 \mathrm{~nm}$. A part of this region was then investigated in a JEOL 2100 TEM at zero tilt, first in the as-deformed condition and then after in-situ annealing. The heating was conducted in multiple steps (see Fig.1), and the microstructure was inspected for each step. Very minor changes in the boundary network were first observed at $260{ }^{\circ} \mathrm{C}$ and became more pronounced with increasing temperature (see Supplementary material). The changes in this region were quantified after final annealing at $400{ }^{\circ} \mathrm{C}$. To facilitate this, the boundary network was manually drawn based on TEM images taken before 
and after annealing, and boundary misorientations were determined using the TKD data. An attempt was made to repeatedly investigate this region by TKD after annealing, but it was not possible to obtain good diffraction patterns due to severe hydrocarbon contamination produced during the first fine-step TKD mapping. It should also be noted that while no recrystallized grains were observed in the investigated region, thicker regions of the annealed foil were found to be partially recrystallized.

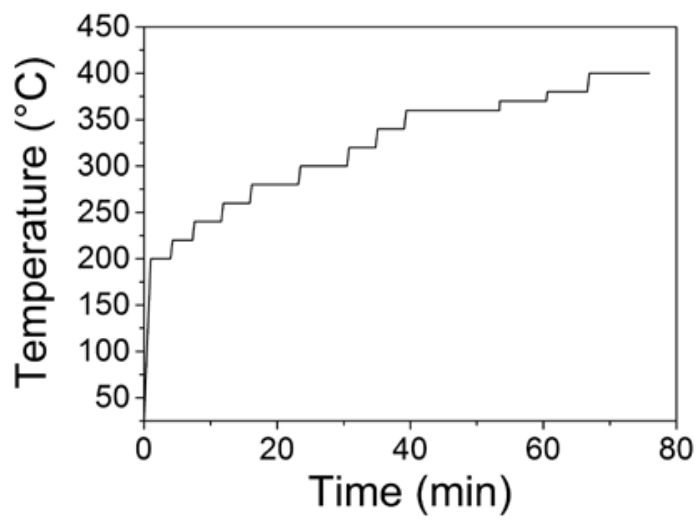

Fig.1. Thermal history of the in-situ annealed foil.

\section{Results}

The microstructure in the as-deformed material (Fig.2a,b) contains lamellar boundaries almost parallel to the $\mathrm{RD}$ and incidental dislocation boundaries (IDBs) within the lamellae. Comparing TKD and TEM images from one region (Fig.2a and Fig.2b, respectively), it is apparent that TKD reveals very fine details of the lamellar structure, where the finest lamella is only $40 \mathrm{~nm}$ thick and the average boundary spacing along the ND is $102 \mathrm{~nm}$. The average spacing between IDBs in this region is $470 \mathrm{~nm}$. The fraction of high angle boundaries (HABs), defined as those with misorientations greater than $15^{\circ}$, is $\sim 50 \%$ as determined by TKD. The analyzed region contains 269 TJs and a small number of four-fold junctions. Following our previous work on Al deformed by equal channel angular extrusion (ECAE) [8], all TJs are categorized into four groups: HHH, HHL, HLL and LLL, where the number of " $\mathrm{H}$ " and "L" corresponds to the number of HABs and low angle $\left(<15^{\circ}\right)$ boundaries (LABs) in each junction. Two dominant groups in the present Ni sample 
are HHL and LLL (118 and 113 TJs, respectively), while there are only 25 TJs of the HHH group and 13 TJs of the HLL group.

Annealing results in reduced dislocation density and in migration of some TJs formed by lamellar boundaries. The latter is illustrated for several junctions in Fig.3c,d. Here it is seen that a number of Y-junctions containing at least two HABs migrate over a distance of $0.1-1.3 \mu \mathrm{m}$ along the RD. The average boundary spacing along the ND increases very little, from $102 \mathrm{~nm}$ to $112 \mathrm{~nm}$.

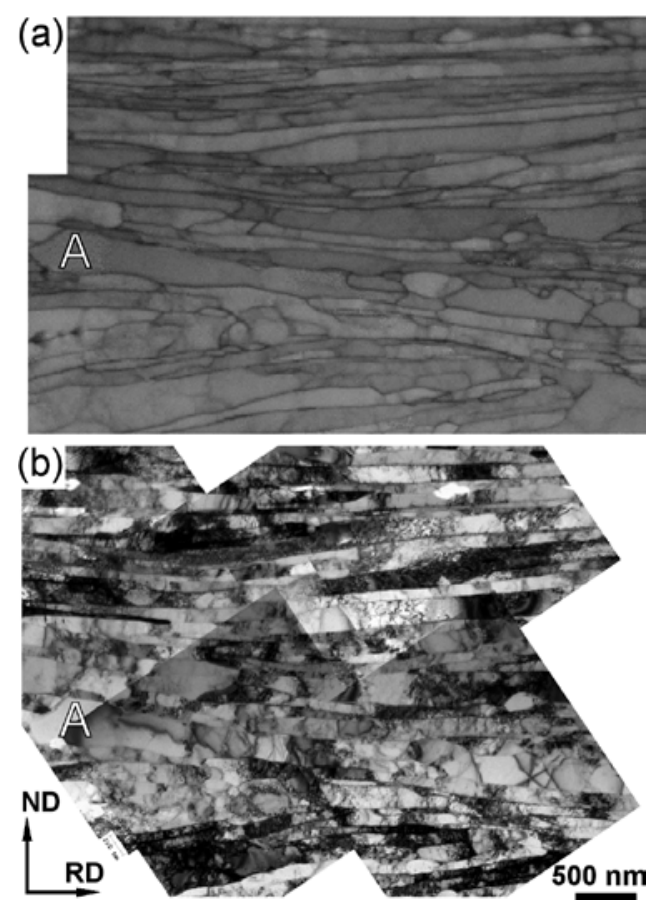

Fig.2. The microstructure in one region of as-deformed Ni investigated using both TKD and TEM: (a) TKD map showing the contrast of Kikuchi bands; (b) TEM image. "A" is a reference label.

For a statistical analysis of TJ motion, all TJs were sorted out into two categories: (i) fastmigrating, defined as junctions migrating over at least $40 \mathrm{~nm}$; and (ii) all other TJs, which were considered slow or stationary. Only 24 TJs (9\% of all TJs) were found to be fast-migrating. There was however a significant difference in the percentage of fast-migrating junctions for the different TJ groups. Approximately a half of the HHH junctions were fast-migrating (see Fig.4). For the HHL and HLL groups, the percentage of fast-migrating TJs was significantly smaller, $8 \%$ and $7 \%$, respectively. We did not find any fast-migrating TJ of the LLL group in the investigated region. 


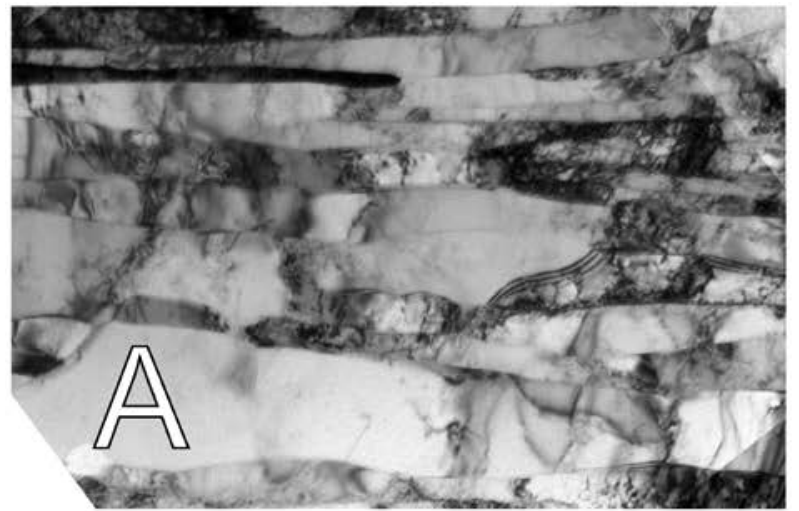

(a)

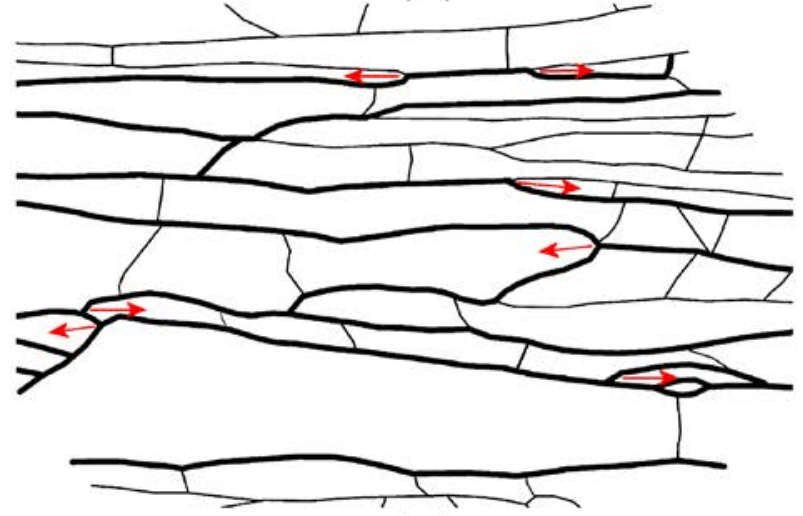

(c)

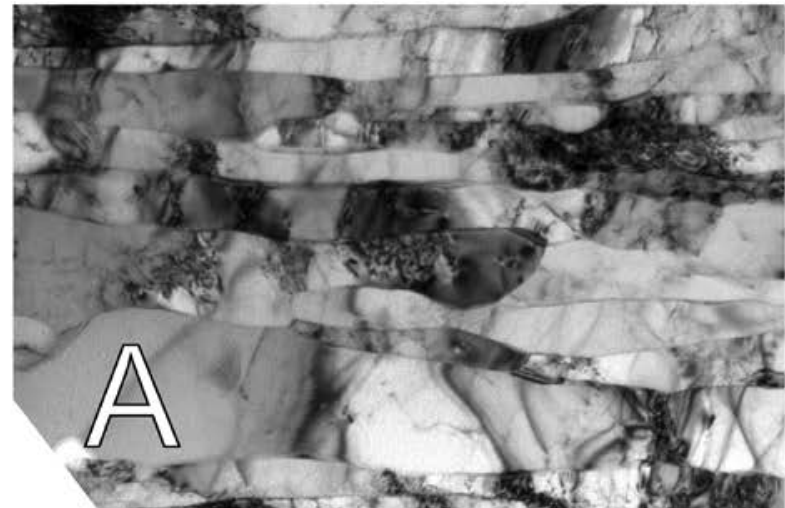

(b)

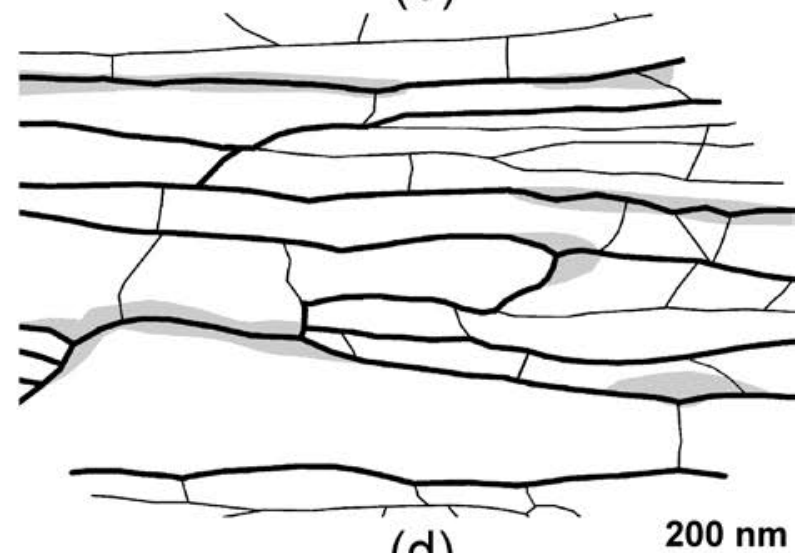

(d)
$200 \mathrm{~nm}$

Fig.3. A fragment of the region shown in Fig.1: (a,c) the as-deformed condition; (b,d) after in-situ annealing. (a,b) are TEM images and (c,d) are drawings showing boundary networks and TJs. Bold and thin lines indicate HABs and LABs, respectively. In (c) arrows indicate the migration direction of Y-junctions. Changes caused by TJ motion are shown in grey in (d).

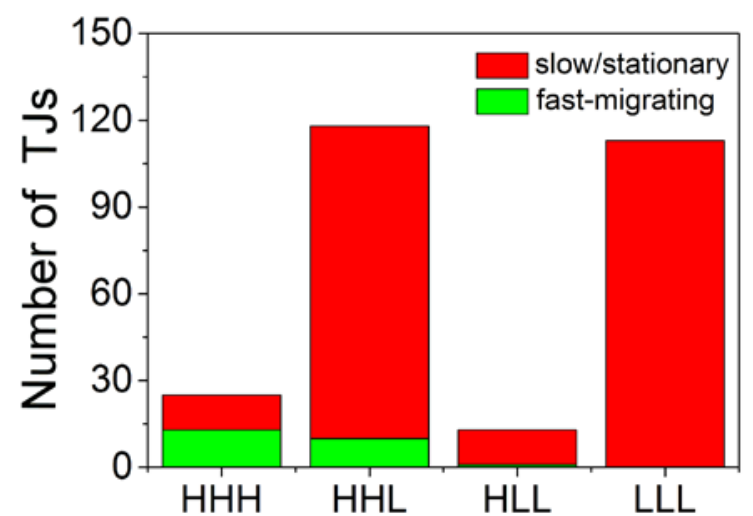

Fig.4. Distribution of TJs in one region investigated both in the as-deformed condition and after insitu annealing. 


\section{Discussion}

One important result obtained in this work is that TJ motion is observed during recovery of heavily deformed Ni. Appreciable TJ motion during in-situ annealing of this material is however revealed only for $9 \%$ of all TJs. Thus, only a small fraction of TJs are fast-migrating junctions, whereas the majority of TJs are fairly stable.

The frequency of fast-migrating junctions for the different TJ groups varies, being much larger for the $\mathrm{HHH}$ group than for the other groups. This result is qualitatively similar to that obtained in a previous study of structural changes during annealing of ECAE-processed Al, for which the percentage of fast-migrating TJs has also been found to decrease with increasing number of LABs in TJs [8]. This dependence reflects lower mobilities of LABs compared to general HABs, and is not surprising. The large difference in the percentage of fast-migrating boundaries between the two materials is however remarkable. In ECAE-processed Al, the overall fraction of fastmigrating TJs (defined in [8] as migrating over at least $100 \mathrm{~nm}$ ) was approximately 40\%. This greater percentage can partly be explained by a much larger frequency of HHH junctions compared to that in the Ni sample. However, fractions of fast-migrating junctions in the Al sample were higher also for each individual group of TJs: 62\%, 30\%, $28 \%$ and $14 \%$ for the HHH, HHL, HLL and LLL groups, respectively [8]. It is possible that morphological differences between the lamellar structures in the ARB Ni sample and deformation structures with less pronounced directionality in the ECAE-processed $\mathrm{Al}$ sample could also contribute to the differences in the extent of $\mathrm{TJ}$ motion in these two samples.

The $\mathrm{TJ}$ motion in the present $\mathrm{Ni}$ sample can also be compared to that in $\mathrm{Al}$ with morphologically similar lamellar structures developed after rolling to a similar strain. TJ motion in $\mathrm{Al}$ results in substantial coarsening, as observed during annealing of TEM foils and bulk specimens [4-7], whereas in Ni the average boundary spacing does not change considerably. This difference in 
the extent of $\mathrm{TJ}$ motion between $\mathrm{Al}$ and $\mathrm{Ni}$ may be related to different IDB spacings in these two materials. Since IDBs can effectively pin TJ motion [6], migrating TJs are less likely to move over large distances in $\mathrm{Ni}$ containing more finely spaced IDBs than in $\mathrm{Al}$, where the average IDB spacing is $780 \mathrm{~nm}$ [4]. More studies of other materials are required to evaluate how materials chemistry, deformation and heat treatment parameters affect TJ motion during recovery in heavily deformed microstructures.

Another significant result obtained in this work is that the characterization approach, in which TKD and TEM are combined for a detailed same-region analysis, is found to be effective. This approach can be used for statistical studies of microstructural evolution during thermomechanical treatments of nanoscrystalline materials.

\section{Conclusion}

Direct evidence of TJ motion in Ni heavily deformed using ARB is provided by examination of one region before and after in-situ annealing in the TEM. By combing crystallographic information obtained using TKD with microstructural changes revealed in TEM images, it is found that during recovery junctions formed by three HABs are more prone to motion than any other TJs. The process of TJ motion in this material results in minor coarsening of the lamellar structure.

\section{Acknowledgements}

The authors acknowledge the support from the Danish National Research Foundation (Grant DNRF86-5) and the National Natural Science Foundation of China (Grant 51261130091). Dr. N. Hansen is acknowledged for useful comments on the manuscript.

\section{References}

[1] O.V. Mishin, A. Godfrey, Metall. Mater. Trans. A, 39 (2008) 2923-2930. 
[2] J. Gubicza, N.Q. Chinh, J.L. Lábár, Z. Hegedűs, T.G. Langdon, Mater. Sci. Eng A, 527 (2010) $752-760$.

[3] F. Lin, Y. Zhang, N. Tao, W. Pantleon, D. Juul Jensen, Acta Mater. 72 (2014) 252-261.

[4] T. Yu, N. Hansen, X. Huang, Proc. Roy. Soc. A 467 (2011) 3039-3065.

[5] T. Yu, N. Hansen, X. Huang, Acta Mater. 61 (2013) 6577-6586.

[6] T. Yu, D.A. Hughes, N. Hansen, X. Huang, Acta Mater. 86 (2015) 269-278.

[7] O.V. Mishin, A. Godfrey, T. Yu, N. Hansen, D. Juul Jensen, IOP Conf.Series: Mater. Sci. Eng. 82 (2015) 012083.

[8] O.V. Mishin, Y.B. Zhang, A. Godfrey, IOP Conf.Series: Mater. Sci. Eng. 89 (2015) 012035.

[9] Y.B. Zhang, O.V. Mishin, N. Kamikawa, A. Godfrey, Proc. Risø Int.Symp. Mater. Sci. 35 (2014) 513-520.

[10] Y.B. Zhang, O.V. Mishin, N. Kamikawa, A. Godfrey, W. Liu, Q. Liu, Mater. Sci. Eng. A 576 (2013) 160-166.

[11] P.W. Trimby, Ultramicroscopy 120 (2012) 16-24.

[12] P.W. Trimby, Y. Cao, Z. Chen, S. Han, K.J. Hemker, J. Lian, X. Liao, P. Rottmann, S. Samudrala, J. Sun, J.T. Wang, J. Wheeler, J.M. Cairney, Acta Mater. 62 (2014) 69-80.

[13] N. Mortazavi, M. Esmaily, M. Halvarsson, Mater. Lett. 147 (2015) 42-45.

[14] D.B. Bober, A. Khalajhedayati, M. Kumar, T.J. Rupert, Metall. Mater. Trans. A, 47 (2016) $1389-1403$. 